Document downloaded from:

http://hdl.handle.net/10251/55068

This paper must be cited as:

Rodrigo Bort, M.; Guillem Sánchez, MS.; Climent, AM.; Pedrón Torrecilla, J.; Liberos Mascarell, A.; Millet Roig, J.; Fernandez-Aviles, F.... (2014). Body surface localization of left and right atrial high-frequency rotors in atrial fibrillation patients: A clinical-computational study. Heart Rhythm. 11(9):1584-1591. doi:10.1016/j.hrthm.2014.05.013.

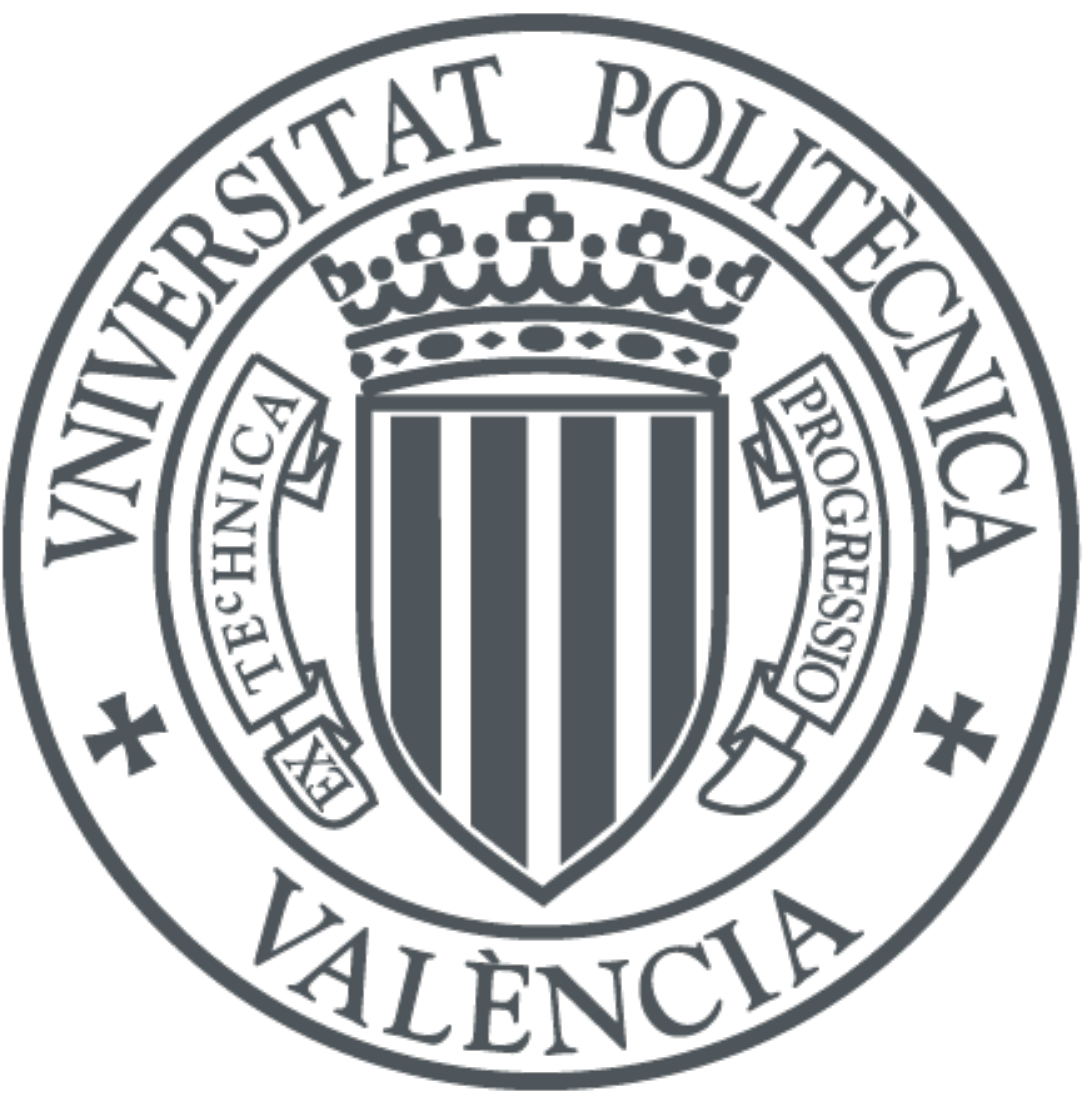

The final publication is available at

http://dx.doi.org/10.1016/j.hrthm.2014.05.013

Copyright Elsevier

Additional Information 


\title{
Body Surface Localization of Left and Right Atrial High Frequency Rotors in Atrial Fibrillation Patients: A Clinical-Computational Study
}

\author{
Body Surface Localization of Atrial Rotors
}

Miguel Rodrigo, M.S. ${ }^{1}$, María S Guillem, Ph.D. ${ }^{1}$, Andreu M Climent, Ph.D. ${ }^{2}$, Jorge PedrónTorrecilla, M.S. ${ }^{1}$, Alejandro Liberos, M.S. ${ }^{1}$, José Millet, Ph.D. ${ }^{1}$, Francisco Fernández-Avilés, M.D., Ph.D. ${ }^{2}$, Felipe Atienza, M.D.,Ph.D. ${ }^{2}$, Omer Berenfeld, Ph.D., F.H.R.S. ${ }^{3}$

${ }^{1}$ Bio-ITACA, Universitat Politécnica de Valencia, Valencia, Spain

${ }^{2}$ Cardiology Department, Hospital General Universitario Gregorio Marañón, Instituto de Investigación Sanitaria Gregorio Marañón, Madrid, Spain

${ }^{3}$ Center for Arrhythmia Research, University of Michigan, Ann Arbor, U.S.A.

Address for correspondence: Maria S. Guillem, ITACA, Universidad Politécnica de

Valencia, Camino de Vera sn, 46022 Valencia (Spain), tel.: +34963877968, email: mguisan@eln.upv.es or Felipe Atienza, Cardiology Department, Hospital General Universitario Gregorio Marañón, Cl Dr Esquerdo 46, 28007, Madrid (Spain), tel.: +34915868281, email: fatienza@secardiologia.es.

\section{Conflicts of interest}

FA served on the advisory board of Medtronic and has received research funding from St. Jude Medical Spain. OB received research support from Medtronic and St. Jude Medical. He is a co-founder and Scientific Officer of Rhythm Solutions, Inc. None of the companies disclosed financed the research described in this manuscript.

\section{Sources of funding}

Supported in part by: the Spanish Society of Cardiology (Becas Investigación Clínica 2009); the Universitat Politècnica de València through its research initiative program; the Generalitat Valenciana Grants (ACIF/2013/021); the Ministerio de Economia y Competitividad, Red RIC; the Centro Nacional de Investigaciones Cardiovasculares (proyecto CNIC-13); the Coulter Foundation from the Biomedical Engineering Department (University of Michigan); the Gelman Award from the Cardiovascular Division (University of Michigan); the National Heart, Lung, and Blood Institute grants (P01-HL039707, P01HL087226 and R01-HL118304), and the Leducq Foundation.

Number of words: $4994 / 5000$ 


\section{ABSTRACT}

Background: Ablation is an effective therapy in atrial fibrillation (AF) patients in which an electrical driver can be identified.

Objective: The aim of this study is to present and discuss a novel and strictly non-invasive approach to map and identify atrial regions responsible for AF perpetuation.

Methods: Surface potential recordings of 14 patients with AF were recorded using a 67-lead recording system. Singularity points (SPS) were identified in surface phase maps after bandpass filtering at the highest dominant frequency (HDF). Mathematical models of combined atria and torso were constructed and used to investigate the ability of surface phase maps to estimate rotor activity in the atrial wall.

Results: The simulations show that surface SPs originate at atrial SPs, but not all atrial SPS are reflected at the surface. Stable SPs were found in AF signals during $8.3 \pm 5.7 \%$ vs. $73.1 \pm 16.8 \%$ of the time in unfiltered vs. HDF-filtered patient data respectively $(p<0.01)$. The average duration of each rotational pattern was also lower in unfiltered than in HDF-filtered AF signals $(160 \pm 43$ vs. $342 \pm 138 \mathrm{~ms}, \mathrm{p}<0.01)$ resulting in $2.8 \pm 0.7$ rotations per rotor. Bandpass filtering reduced the apparent meandering of surface HDF rotors by reducing the effect of the atrial electrical activity taking place at different frequencies. Torso surface SPS representing HDF rotors during AF were reflected at specific areas corresponding to the fastest atrial location.

Conclusion: Phase analysis of surface potential signals after HDF-filtering during AF shows reentrant drivers localized to either the LA or RA, helping in localizing ablation targets.

KEY WORDS: atrial fibrillation, electrocardiography, mapping, atrial rotor, body surface potential mapping. 
NON-STANDARD ABBREVIATIONS AND ACRONYMS

65 HDF - Highest Dominant Frequency

SP - Singularity Point

LA-HDF - Left Atria Highest Dominant Frequency

RA-HDF - Right Atria Highest Dominant Frequency

BSPM - Body Surface Potential Mapping 


\section{INTRODUCTION}

Recent invasive [1-2] and noninvasive [3-6] mapping studies of human AF have demonstrated a variety of spatiotemporal patterns of activation, including high frequency sites, rotors and focal discharges during human AF. Arguably, the identification of the AF mechanism in each patient may help selecting the best therapy or ablation strategy to deliver to that individual patient. However, how to map effectively and determine the specific mechanisms of AF maintenance in individual patients is still elusive [7].

It has recently been shown that non-invasive mapping allows the identification of high dominant frequency (DF) atrial sources during human AF [5]. However, the ability of surface mapping data to determine the specific activation patterns underlying the fast activation that characterize AF (i.e. focal vs. re-entrant activity) has not been studied before. The aim of this study is to investigate the potential use of surface mapping recordings for the estimation of propagation patterns during AF on an individual patient basis. 


\section{METHODS}

\section{Patients and body surface potential recording}

The 14 patients included in this study were admitted for ablation of drug-refractory paroxysmal and persistent AF (see online Table 1). All patients gave informed consent. The protocol was approved by the Institutional Ethics Committee. In patients arriving in sinus rhythm, AF was induced by electrical burst pacing.

Intracardiac electrograms (EGMs) were sequentially obtained from both atria during the procedure [5]. Power spectral density of EGMs was computed to determine the local DFs in each atrium.

Surface electrocardiograms (ECGs) were recorded simultaneously with the intracardiac EGMs using a grid of 67 electrodes on a vest covering the torso (Figure S1A). Ventricular activation was removed by administration of a central venous bolus of adenosine (12-18 $\mathrm{mg})$ and 4-seconds segments of surface ECGs surrounding the longest RR interval were used for the analysis. Acquired surface potentials on the entire torso were baseline-subtracted and low-pass filtered at $30 \mathrm{~Hz}$ [3]. Power spectral density of all signals was computed to determine the local DFs and their distribution on the body surface [5].

Surface ECGs were then filtered at the highest DF (HDF) found on the torso surface or at the highest DF found at either left atrium EGMs (LA-HDF) or right atrium EGMs (RA-HDF) by using a $2 \mathrm{~Hz}$ bandwidth band-pass filter (see Online supplement).

\section{Computational models of the atria and torso}

To guide processing and interpretation of the recorded body surface potentials we simulated the electric potentials behavior on a computerized model of the atria within the torso during different impulse propagation patterns. This approach will enable investigating for the first time the manifestation of atrial reentrant activity on both the internal volume and the torso surface. We constructed a generic atria-torso model consisting of a spherical shell of active 
tissue, representing the atria, within a passive torso modeled as a uniform volume conductor bounded by a spherical surface with no-flux conditions (see Online supplement).

The electric potential resulting from the wave propagation simulated on the atrial sphere was studied everywhere on 20 concentric spheres by using the Boundary Element Method [8]. The potentials on the external sphere, defined as the torso surface, and in the internal layers were analyzed to characterize the time-course of the potential distribution everywhere; in particular, the patterns and time-course of the filaments resulting from atrial rotors were characterized.

\section{Phase singularity and filaments}

120 Phase maps on the torso surface of patients and in each concentric layer in the computer models were obtained from the potential phase signal of each node by the Hilbert transform [9]. A singularity point (SP) was defined as the point in a phase map which is surrounded by phases from 0 to $2 \pi$. Only those SPs that were present for the duration of at least one full rotation were considered (see Online supplement).

125 A filament was defined as the connection between SPs across spherical layers at a given time. Only filaments that complete at least one rotation on the outermost surface were considered.

\section{Statistical analysis}

The t-test was used to evaluate the statistical significance between continuous paired or unpaired variables, and statistical significance was considered for $p<0.05$. All data are reported in mean $\pm S D$. 


\section{RESULTS}

\section{Surface mapping of atrial activity during AF}

Surface phase maps of the unipolar voltage time-series recorded during AF show unstable patterns, as can be appreciated by the transient SPs seen in the maps from a sample patient presented in Figure 1A, as opposed to stable rotational patterns observed during atrial flutter (Figure S1). Long-lasting SPs were rarely observed during AF without band-pass filtering and those observed tended to drift erratically large distances in short time. However, after band-pass filtering of the potential signal around the HDF $(6.8 \mathrm{~Hz})$, surface phase maps showed more stable SPs for the same AF episode (Figure 1A). In Panel B the arrows connecting sequential activations in ECGs recorded around the SP in Figure $1 \mathrm{~A}$ show a clear reentrant pattern which following HDF-filtering transformed into long-lasting rotational patterns with stable SPs. Considering data from all patients, stable SPs were found in unfiltered AF signals during $8.3 \pm 5.7 \%$ of the time vs. $73.1 \pm 16.8 \%$ following HDF-filtered signals $(p<0.01)$. The average SPs duration concomitantly increased following the HDFfiltering ( $160 \pm 43$ vs. $342 \pm 138 \mathrm{~ms}, \mathrm{p}<0.01)$. At an average HDF of $9.2 \pm 2.3$ (BSPM) or $9.3 \pm 2.0$ $(E G M) \mathrm{Hz}$, the latter corresponds to an average of $2.9 \pm 0.7$ continuous rotations per SP observed in our cohort of patients. Many observed SPs drift and appear or disappear on the borders of the surface mapped area, or in the beginning and end of the periods analyzed, thus this average number of rotations represents a lower limit for their life span. Indeed, Figure $1 \mathrm{C}$ and the Online Movie 1 show a rotor in the middle of the mapped area at the beginning of the period analyzed that after about $1400 \mathrm{~ms}$ disappears at the lower boundary of the area. After a period of fuzzy SP behavior in the posterior torso (see Movie 1) for about $330 \mathrm{~ms}$ an SP appears and remains in the mapped area for the rest of the analyzed period. This case shows that the actual life span of rotors could be longer than the conservative average life-span we calculate. Further evidence of atrial rotor drifting is provided by the simultaneous EGM recorded at the highest DF site (Figure 1E), which is unstable in intervals 
1-4 and monomorphic at interval $4-5$, which is consistent with the observed drifting on the torso surface.

\section{Simulations to understand HDF band-pass filtering of AF patterns}

The finding that the dynamics of the body surface SPs dramatically depends on the HDFfiltering raises questions regarding the phase maps interpretation. Unfortunately, the ability to collect simultaneously electrical data in the atria and inside the torso volume and surface to make inferences between atrial activity and its manifestation on the body surface is limited, leading us to rely on computer simulations for guidance.

In Figure 2 a simulation with a LA-RA atrial model and the multi-spheres torso model is depicted. In this model there is a single functional rotor in the LA hemisphere turning at 7.2 $\mathrm{Hz}$ while the RA hemisphere is passively activated at lower a frequency $(3.9 \mathrm{~Hz})$. Figure $2 \mathrm{~A}$ shows the phase maps of three concentric layers between the epicardium (left) and the torso surface (right) at various times. The phase maps become smoother toward the torso surface, reflecting the low-pass filter effect of the passive torso volume on the extracellular potentials. In the epicardial layer there is one stable SP at the location of the functional rotor (LA) that appears at similar positions in the outer layers, and another SP at the less stable wavebreak location at the interface between the faster LA and the slower RA. Azimuth and elevation of SPs detected on the surface are not preserved across layers, so the filament arising from the LA rotor exhibits a deflection in its trajectory to the torso. The deflection angle of the filaments before HDF-filtering is not stationary over time and, instead, the filament trajectory describes a cone. However, Figure 2B shows that HDF-filtering significantly reduces the filaments' deflection and stabilizes them to follow a straight path from the epicardium to the surface. We hypothesize that the HDF-filtering minimized the influence of the atrial activity that is not activated at the HDF and thus stabilized the filament. To corroborate such hypothesis we sum all the RA dipoles into a single equivalent dipole and plot its vector projection on the RA-LA interface $(Y, Z)$ plane (Figure 2C). In addition we plot the trajectory 
of the external SP originated by the LA rotor projection on the same $(Y, Z)$ plane. In Figure $2 \mathrm{D}$ we notice the match in the time course and rotation frequency between the RA equivalent dipole rotation and the SP trajectory. Accordingly, we conclude that SPs arising at the interface between LA and RA as a consequence of abrupt changes in propagation direction that reached the outermost layer disappear after HDF-filtering since their activation frequencies did not match with the HDF. In addition, we notice that a mirror filament appears with opposite direction and chirality as compared to the true rotor originating at the SP on the LA epicardium. Indeed, following the attenuation of RA activation frequencies, potentials on the RA hemisphere are caused by the rotating electrical activity on the LA and observed from a contralateral point of view (see Figure S2).

We further explore the behavior of filaments under more complex wave patterns; in Figure 3 we analyze simulation results from a 50\% LA area and 50\% fibrotic area model. In this case epicardial activity consists of a LA rotor with a stable SP and a very disorganized activity with several unstable SPs in the fibrotic area (Figure $3 \mathrm{~A}$ ). In Figure $3 \mathrm{~B}$ most of the filaments originate at SPs involving a small piece of fibrotic tissue but do not originate at the driving rotor. As can be seen, the spatial smoothing stabilizes filaments and eliminates all but a single filaments' pair reaching the surface. To understand how the filaments originating at the epicardium disappear inside the torso, we color coded them according to the chirality of the SPs. Figure $3 \mathrm{C}$ clearly shows that filaments are continuous and do not vanish inside the passive volume conductor, rather a filament does not reach the surface once it is joining its counter-rotating neighbor. Overall, that mutual cancelation of filament pairs reduces the average number of SPs at increasing distances from the epicardium (Figure 3D). At the outermost layer, only two SPs can be observed: one which is the "true" SP and another that we term the "mirror" SP and appears following the extinction of SPs at the fibrotic area and extension of the stable LA rotor filament to the contralateral aspect of the torso. Mutual cancellation of filaments can indeed result in a failure to detect "true" SPs originating at small atrial areas (Figure S3); an effect that may be counteracted by the HDF-filtering. 
Indeed, the discrimination between true and mirror rotors can be performed based on the spectral properties of surface recordings at the HDF. In Figure 4A we show a surface phase map with 2 SPs that resulted from HDF-filtering of a simulation with only one stable rotor. The SP labeled T is the end point of the filament that connects directly to the epicardial SP (hence the label "true" SP); the SP labeled $M$ is the surface end point of a filament originating at the epicardial wall contralateral to the stable rotor (hence the term "mirror" SP; see Figure S2). On the phase map these SPs seem undistinguishable; however the spectral power at the rotor frequency band is maximal at the "true" SP. Panel B of Figure 4 shows a similar analysis in a patient. The patient's surface phase map shows 2 SPs and the traces below the map show that while in one SP location the maximal power is at the HDF, in the other SP the power at that frequency is much less, indicating that the former is the true SP and the latter is the mirror SP.

\section{Centers of rotational activity in human AF}

True SPs detected, defined as those with at least $60 \%$ of their spectral content at the HDF band (Figure 4), tended to concentrate at certain torso areas related with the DF distribution in the atria [5]. In Figure 5A, the trajectory of a surface SP that drifted during 2 seconds on the posterior torso of a patient following LA-HDF filtering (band-pass filtering at the HDF found in simultaneous left intracardiac EGMs recordings) is depicted (see Online movie 1, from $t=1850$ to $t=3850$ ). In Figure $5 B$ the trajectory of a SP that drifted during $500 \mathrm{~ms}$ on the right anterior torso in another patient after RA-HDF filtering is shown (see Online movie 2). In Figure 5C, the 2-dimensional histogram of "true" SP locations after LA-HDF filtering in patients with an inter-atrial DF gradient $>1 \mathrm{~Hz}(n=10)$ shows a predominant location of SPS on the posterior torso. The 2-dimensional histogram of "true" SP locations after RA-HDF filtering in patients with inter-atrial DF gradient shows a predominant localization on the right anterior torso, Figure 5D. The locations of the maximal numbers of true LA or RA SPs (dark red regions) are shown in Panels $C$ and $D$ to reside well within the areas demarcated by the 
HDFs originating either at the LA or RA, respectively, based on our previous surface-atrial DF distribution correlation study [5]. 


\section{DISCUSSION}

In this study we show that phase maps of surface potentials during AF after HDF-filtering allow observing reentrant patterns with spatiotemporal stability. In contrast, the unfiltered surface phase maps display unstable reentrant patterns. The short-lived and unstable surface reentrant activity in the non-filtered data is suggested by computer simulations to result from superposition of irregular electrical activity at frequencies other than the HDF which may mask the presence of the more stable reentrant activation.

\section{Rotors and AF maintenance in humans}

250 Circus propagation was proposed as a possible mechanism for perpetuation of AF as early as in the 1920 [10] and gained relevance after experimental demonstration of functional rotors maintaining AF by optical mapping in isolated sheep heart preparations [11]. However some studies continue advocating for the alternative mechanism of multiple wavelets as the predominant mechanism underlying AF maintenance [12]. In humans, evidence for stable rotors as a mechanism of AF maintenance was until recently sporadic and indirect [13] and also questionable [14]. Narayan et al. reported recently that rotor activity is detectable by panoramic intracardiac mapping in about $70 \%$ of 98 out of $101 \mathrm{AF}$ patients and brief ablation at the centers of those rotors was effective in terminating or slowing the arrhythmia [2]. Although the interpretation of the mapping and ablation results obtained with the basket catheter are still somewhat controversial [7] they constitute strong evidence for the major role of rotors in maintaining AF. The results presented in this study are consistent with that latter evidence: the BSPM is detecting rotor activity also in about $70 \%$ of the time during AF. Our study further demonstrates however, that the rotor activity is the fastest activity in the patients we studied. 
Previous high-density surface mapping of atrial propagation patterns showed that the presence of surface reentries was infrequent and accounted for few consecutive rotations [34]. Results from our mathematical simulations suggest that the ability of raw surface potentials in detecting existing rotors is compromised by (i) the organization degree of areas activated at lower rates than the rotor and (ii) the amount of myocardial tissue activated at the same rate as the rotor. Interestingly, computer simulations demonstrate that the far field influence of areas with low organization could produce less distortion on SPs torso projection than areas with higher organization. However, activity of rotors covering a small atrial portion may be masked by the electrical activity of the rest of the atria, even if it is a highly disorganized activity (see Figure S3). Band-pass filtering of ECG signals at the activation frequency of rotors with a driving role (i.e., a mother rotor) would accentuate the electrical activity taking place at the passing frequency band by attenuating the influence of electrical activity occurring at other rates and therefore, allows the detection of atrial SPs at the nearest projected location on the surface. Altogether, we propose that band-pass filtering may allow observing rotational patterns in cases of rotor involvement of large tissue areas and/or a disorganized activity of areas not involved in the rotation maintenance.

Previous studies in which epicardial potentials were reconstructed trough the inverse solution formalism reported that rotor activity was rarely seen and account for only $15 \%$ of patterns observed [4], although consistently observed in some selected patients [6]. These results are consistent with our observations in raw ECG signals from patients, with $8 \%$ of processed maps presenting stable rotors with more than one consecutive rotation. Our simulations results show that surface activation patterns do not preserve either the global activation pattern nor the shape of activation wavefronts. In fact, patterns observed on the torso surface represent a spatially smoothened version of epicardial propagation. A novel observation in our simulations analysis is that the filaments of epicardial rotors with opposing chiralities, particularly nearby rotors, may join inside the torso and thus those rotors are not detectable on its surface at all, further smoothing complex patterns and importantly 
suggesting that their epicardial reconstruction through the inverse solution is not attainable. Thus, the ability of surface ECG data to accurately retrieve complex epicardial propagation AF patterns from the patterns on the torso needs to be further explored.

\section{Phase map analysis in surface ECG signals}

Phase map analysis applied to surface signals has been proven useful for displaying the propagation pattern projected on the torso. Atrial rotor patterns with transmural filament, as simulated in our models here, would always produce two contralateral axes of extracellular potential rotation, that is, filaments, in the volume conductor (i.e. one pointing into the atrial cavity and the other out of the cavity). The intersections of those filaments with the torso surface thus produce two SPs: one SP at the nearest point on the surface from the epicardial SP (or "true" SP) and another "mirror" SP at the opposite side. Surface phase maps in patient data do not always reflect two simultaneous SPs because of an incomplete (not geometrically closed) mapping area. Since phase maps are insensitive to the signal power at different locations, both "true" and "mirror" SPs look similar. However, spectral analysis at the location of SPs may allow to discriminate between "true" and "mirror" SPS based on the power content at the HDF.

\section{Rotors and dominant frequencies in human AF}

Optical mapping both in Langendorff-perfused animal hearts during fibrillation [15] and monolayers of neonatal rat ventricular myocytes [16] has consistently shown that there is a hierarchical distribution of DF in fibrillating cardiac tissue whereby the fastest regions act as sources driving the slower ones. These high-frequency sources sustain in most cases the AF activity of both atria and may harbor functional rotors. These findings are consistent with electrogram analysis of human AF in which this hierarchical DF pattern has been linked to the existence of drivers at high-frequency sites $[1,13]$ as well as drifting rotors at sites with transient electrogram fractionation [17]. Besides, it has been found that adenosine infusion during AF, increases local DFs, particularly at fastest activating sites at baseline, highlighting 
the hierarchical organization in the DF and rate of activation of different regions in the atria [13].

The hierarchical DF patterns can also be detected from surface recordings so that HDF regions can be identified and related to intracardiac DFs and DF gradients [5]. Our results suggest that human AF is driven by rotors most of the time (73\%) as they could be detected after HDF-filtering. The possible reasons for the absence of rotors during the other $27 \%$ of the time are: (i) a rotational pattern was present outside the mapped area, (ii) the atrial rotor generates an un-detectable signal on the surface, or that (iii) AF was driven by ectopic foci.

\section{Study Limitations}

We cannot conclusively confirm that rotational patterns observed in patients correspond to actual atrial rotors since we do not have simultaneous epicardial and/or endocardial panoramic data. However, we have made use of mathematical models to demonstrate that in case rotors are present during AF on the surface, as we demonstrate in the patients, they are generated by atrial rotors. Although the models of the atria-torso system were simple spherical models, these simple models contain the active and passive volume conductor components needed to gain insight into the mechanisms of visual rotor stabilization by HDFfiltering. Insertion of inhomogeneities in the torso model such as lungs do not alter the main conclusions reached here, since they produce a reduction on the surface voltages but not in their morphology (see Figure S4). In the human torso as well as in a more realistic anatomical model, the projection of epicardial potentials on the surface of the torso will certainly be altered in comparison to those in the spherical models used here and, in particular, certain atrial areas could be masked, but the effects of the volume conductor and band-pass filtering shown here on the torso filaments and the surface SPs should hold. Finally, to reduce ventricular activity from the surface recordings and highlight HDF values our AF mapping study was performed in the presence of adenosine which may alter 
fibrillatory activity and the spatial domain of the rotors in the atria, and potentially alter the 345 sensitivity for their detection of the surface. 


\section{CONCLUSION}

Our clinical-computational study suggests that the body surface data on wavebreaks during AF is incomplete, but it contains features that can be linked to reentrant drivers of AF. Narrow band-pass filtering allows selecting the electrical activity projected on the torso at the HDF, which stabilizes the projection of rotors that potentially drive AF on the surface. Phase maps of HDF-filtered surface ECG recordings may allow the noninvasive localization of atrial re-entries during AF, enabling further physiologically-based rationale for considering the constraints of the inverse solutions. This approach may, therefore, help in planning and performing ablation procedures, decreasing the amount of time required for the search of AF drivers.

\section{ACKNOWLEDGMENTS}

We appreciate the discussions with Dr. Christian Zemlin. 


\section{REFERENCES}

1. Atienza F, Almendral J, Jalife J, Zlochiver S, Ploutz-Snyder R, Torrecilla EG, Arenal A, Kalifa J, Fernández-Avilés F, Berenfeld O: Real-time dominant frequency mapping and ablation of dominant frequency sites in atrial fibrillation with left-to-right frequency gradients predicts long-term maintenance of sinus rhythm. Heart Rhythm 2009; 6:33-40.

2. Narayan SM, Krummen DE, Shivkumar K, Clopton P, Rappel WJ, Miller JM: Treatment of atrial fibrillation by the ablation of localized sources: CONFIRM (conventional ablation for atrial fibrillation with or without focal impulse and rotor modulation) trial. J Am Coll Cardiol 2012; 60:628-636.

3. Guillem MS, Climent AM, Castells F, Husser D, Millet J, Arya A, Piorkowski C, Bollmann A: Noninvasive mapping of human atrial fibrillation. J Cardiovasc Electrophysiol 2009; 20:507-513.

4. Cuculich PS, Wang Y, Lindsay BD, Faddis MN, Schuessler RB, Damiano RJ Jr, Li L, Rudy Y: Noninvasive characterization of epicardial activation in humans with diverse atrial fibrillation patterns. Circulation 2010; 122:1364-1372.

5. Guillem MS, Climent AM, Millet J, Arenal A, Fernández-Avilés F, Jalife J, Atienza F, Berenfeld O: Noninvasive localization of maximal frequency sites of atrial fibrillation by body surface potential mapping. Circ Arrhythm Electrophysiol 2013; 6:294-301. 
6. Haissaguerre M, Hocini M, Shah AJ, Derval N, Sacher F, Jais P, Dubois R: Noninvasive panoramic mapping of human atrial fibrillation mechanisms: a feasibility report. J Cardiovasc Electrophysiol 2013; 24:711-717.

7. Berenfeld $\mathrm{O}$, Oral $\mathrm{H}$ : The quest for rotors in atrial fibrillation: different nets catch different fishes. Heart Rhythm 2012; 9:1440-1.

8. Horácek BM, Clements JC: The inverse problem of electrocardiography: a solution in terms of single- and double-layer sources of the epicardial surface. Math Biosci 1997; 144:119-154.

9. Zlochiver S, Yamazaki M, Kalifa J, Berenfeld O: Rotor meandering contributes to 400 irregularity in electrograms during atrial fibrillation. Heart Rhythm 2008;5:846-54

10. Lewis T: Oliver-Sharpey Lectures: On the nature of flutter and fibrillation of the auricle. $\mathrm{Br}$ Med J 1921; 1:590-593. of atrial fibrillation. Cardiovasc Res 2002; 54:204-216. 
12. Lee S, Sahadevan J, Khrestian CM, Durand DM, Waldo AL: High density mapping of atrial fibrillation during vagal nerve stimulation in the canine heart: restudying the Moe hypothesis. J Cardiovasc Electrophysiol 2013; 24:328-335.

13. Atienza F, Almendral J, Moreno J, et al: Activation of inward rectifier potassium channels accelerates atrial fibrillation in humans: evidence for a reentrant mechanism. Circulation 2006; 114:2434-2442.

14. de Groot NM, Houben RP, Smeets JL, Boersma E, Schotten U, Schalij MJ, Crijns H, Allessie MA: Electropathological substrate of longstanding persistent atrial fibrillation in patients with structural heart disease: epicardial breakthrough. Circulation 2010; 122:167482.

15. Mansour M, Mandapati R, Berenfeld O, Chen J, Samie FH, Jalife J: Left-to-right gradient of atrial frequencies during acute atrial fibrillation in the isolated sheep heart. Circulation 2001; 103:2631-2636.

16. Campbell KF, Calvo CJ, Mironov S, Herron T, Berenfeld O, Jalife J: Spatial gradients in action potential duration created by regional magnetofection of hERG are a substrate for wavebreak and turbulent propagation in cardiomyocyte monolayers. J Physiol 2012; 590:6363-6379.

430 17. Atienza F, Calvo D, Almendral J, Zlochiver S, Grzeda KR, Martínez-Alzamora N, González-Torrecilla E, Arenal A, Fernández-Avilés F, Berenfeld O: Mechanisms of 
fractionated electrograms formation in the posterior left atrium during paroxysmal atrial fibrillation in humans. J Am Coll Cardiol 2011; 57:1081-1092. 


\section{FIGURE LEGENDS}

Figure 1. Surface phase maps during AF. (A) Surface phase maps at three selected times for unfiltered (left) and for HDF-filtered (right) surface potentials. (B) ECGs at positions 1-6 marked in (A) before and after HDF-filtering and Power Spectral Density (PSD) for unfiltered ECGs. Time marker at 2656 ms corresponds to the top map in the HDF-filtered data in (A). (C) PSs trajectories on the torso surface during a 3-sec long AF. (D) Percentage of time with rotors (up) and rotor duration (down) in surface phase maps from unfiltered and HDF-filtered surface potentials over the entire cohort. (E) Electrogram recorded at the highest DF site in the atria (RSPV) simultaneously to the surface recordings.

Figure 2. Epicardial and transition to surface phase maps during AF in a 50\% LA - 50\% RA atrial model. (A) Phase maps at 4 time instants (top to down) in 3 concentric layers at increasing distances from the epicardium (left to right) and after HDF-filtering of surface potentials. (B) Phase map of epicardial sphere and temporal distribution of filaments for unfiltered potentials and for HDF-filtered potentials. (C) Tracking of the dipole direction on the RA on the $\mathrm{YZ}$ plane (virtual septum plane) and tracking of the surface rotor than arises from the LA without HDF-filtering. (D) Temporal evolution and spectral distribution of $Y$ coordinate for (C).

Figure 3. Epicardial and transition to surface phase maps during AF in a $50 \%$ LA - 50\% fibrosis atrial model. (A) Phase maps at 4 time instants (top to down) in 4 concentric layers at increasing distances from the epicardium (left to right). (B) Phase map of epicardial sphere and temporal evolution of filaments for unfiltered potentials. (C) Phase map of epicardial sphere and distribution of filaments (clockwise rotations in blue, counterclockwise rotations in red) inside the torso during $10 \mathrm{~ms}$. (D) Average number of rotors per frame. 
Figure 4. Discrimination of true and mirror SPs at surface phase maps in a simulated and human AF. (A) Surface phase map in 50\% LA - 50\% fibrosis atrial model and power spectra of surface potentials at the true rotor location (blue, T) and at the mirror rotor location (red, M). (B) Surface phase map after HDF-filtering from patient \#11 during AF and power spectra of surface potentials prior to the filtering at the true rotor location (blue, $\mathrm{T}$ ) and at the mirror rotor location (red, M).

Figure 5. Spatial distribution of surface rotors in human AF. (A) Phase map and rotor tracking (blue scale) after LA-HDF filtering in an LA-fastest patient. (B) Phase map and rotor tracking (blue scale) after RA-HDF filtering in an RA-fastest patient. (C) Histogram of the rotor position for all rotors detected in patients with an inter-atrial DF gradient after LA-HDF filtering. LA-detected region is outlined with a dotted line. (D) Histogram of the rotor position for all rotors detected in patients with an inter-atrial DF gradient after RA-HDF filtering. RAdetected region is outlined with a dotted line. 


\section{FIGURE 1}

A

Unfiltered phase map
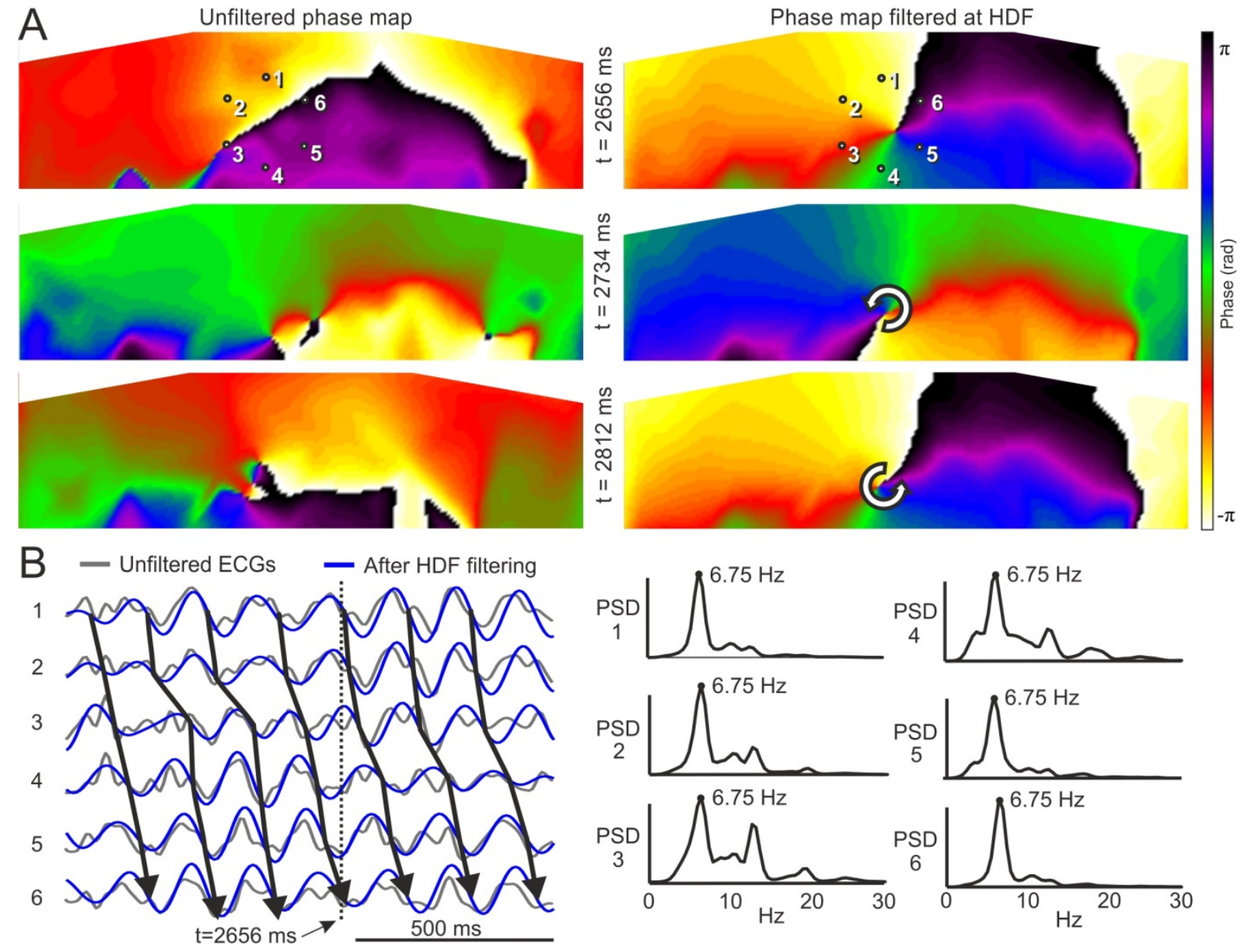

$c$
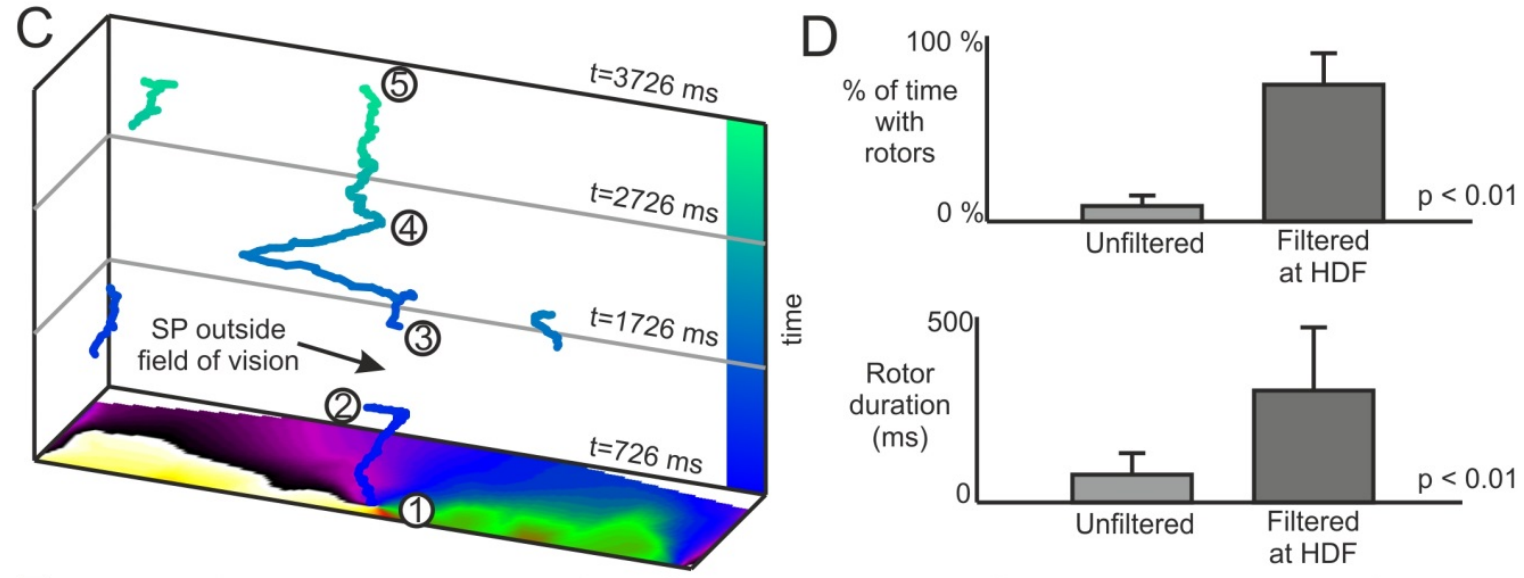

E (1) (2) (3)

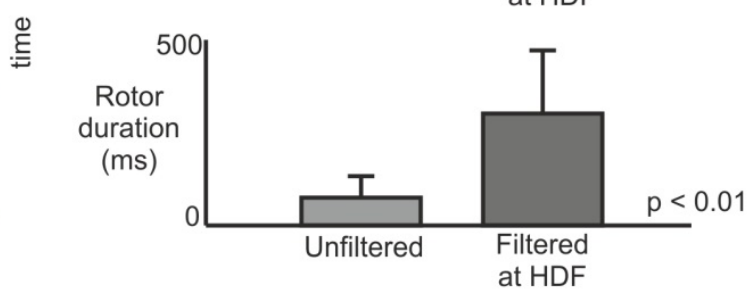

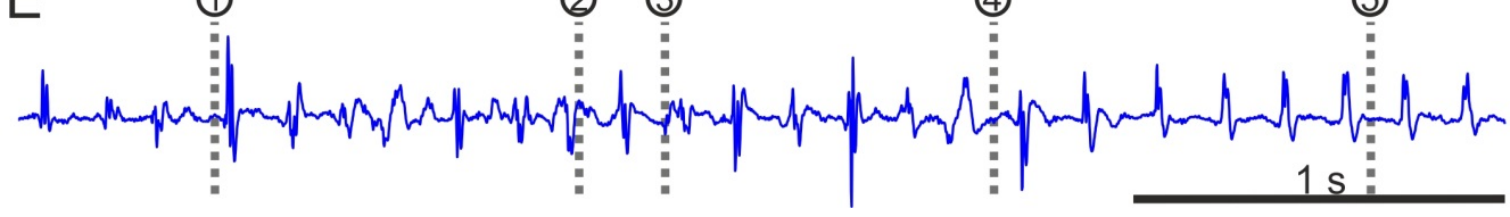




\section{FIGURE 2}
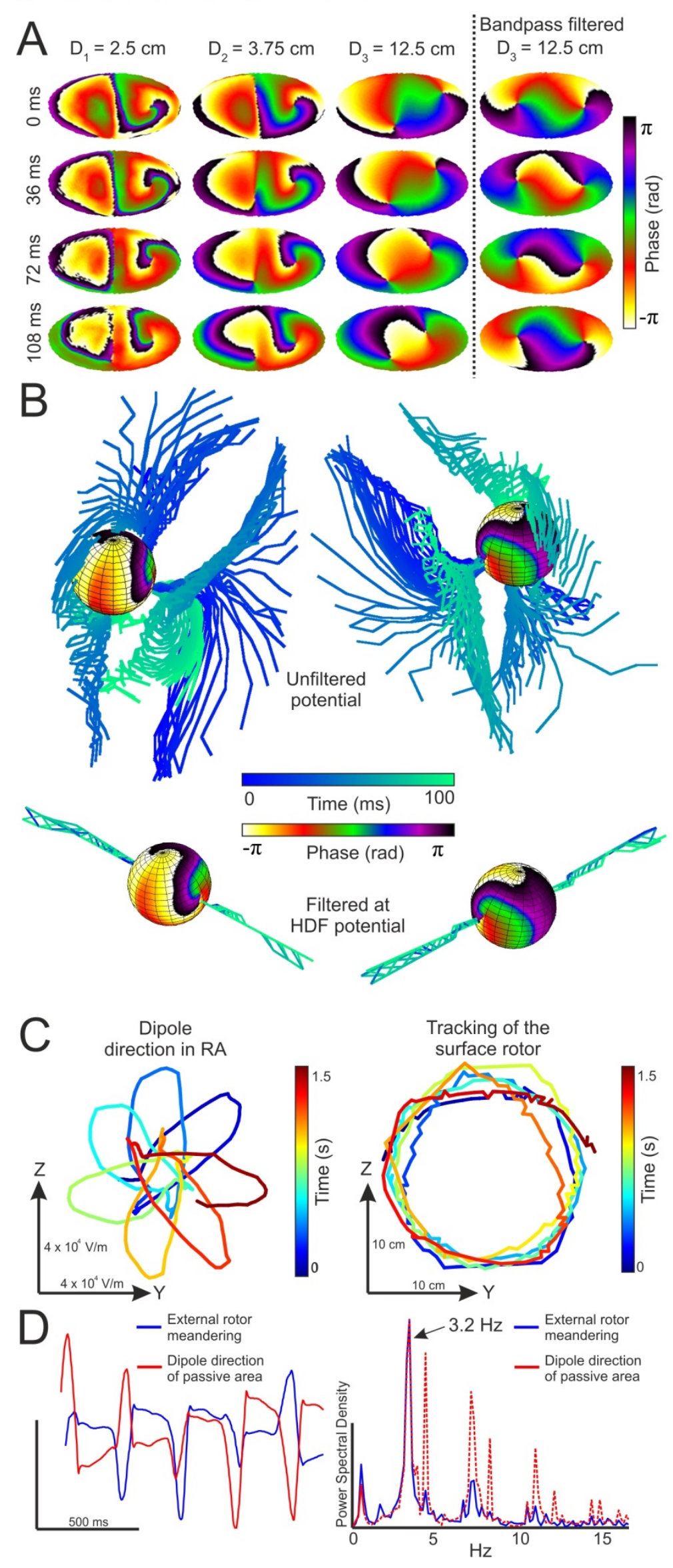


\section{FIGURE 3}
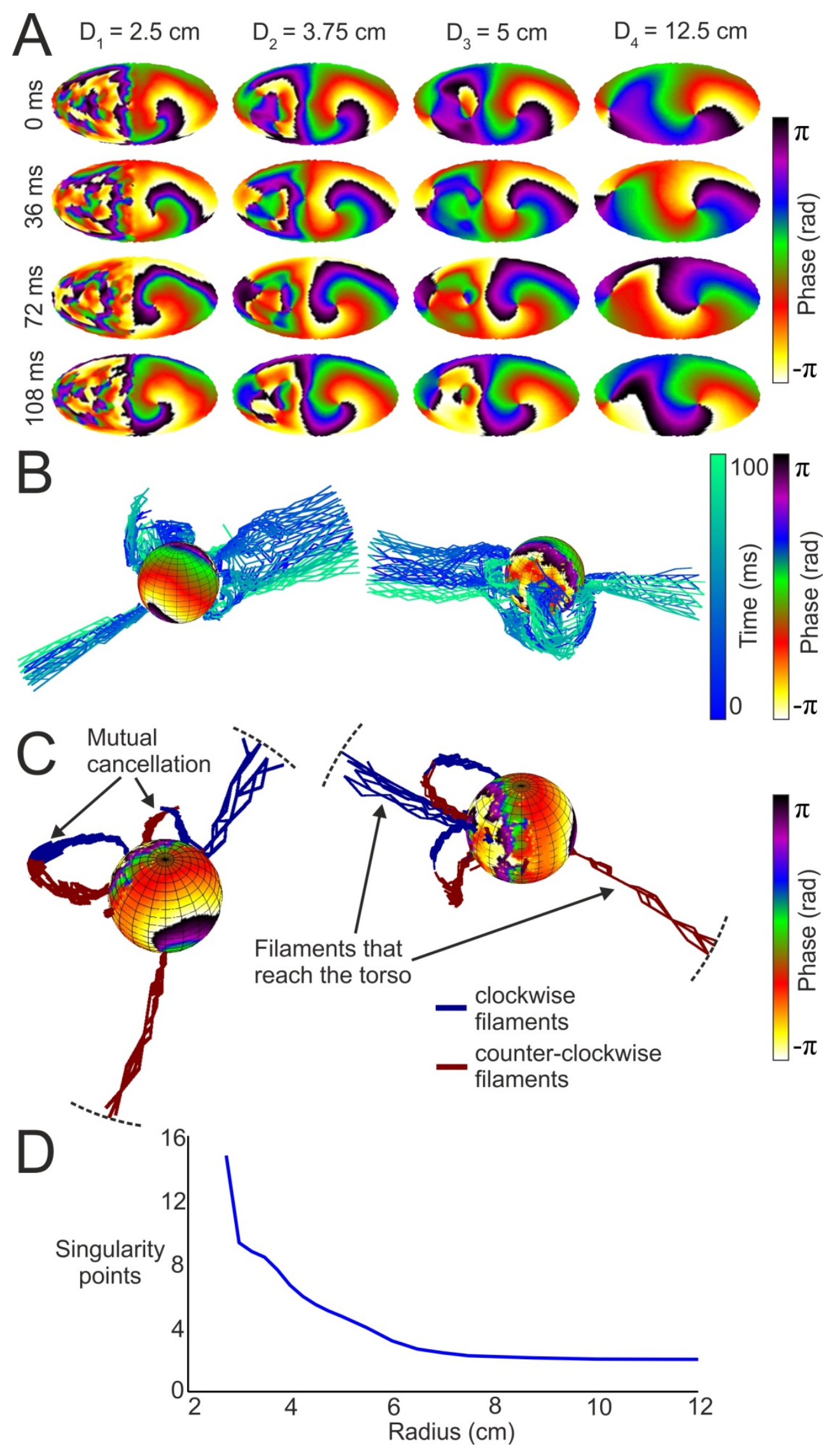


\section{FIGURE 4}

A
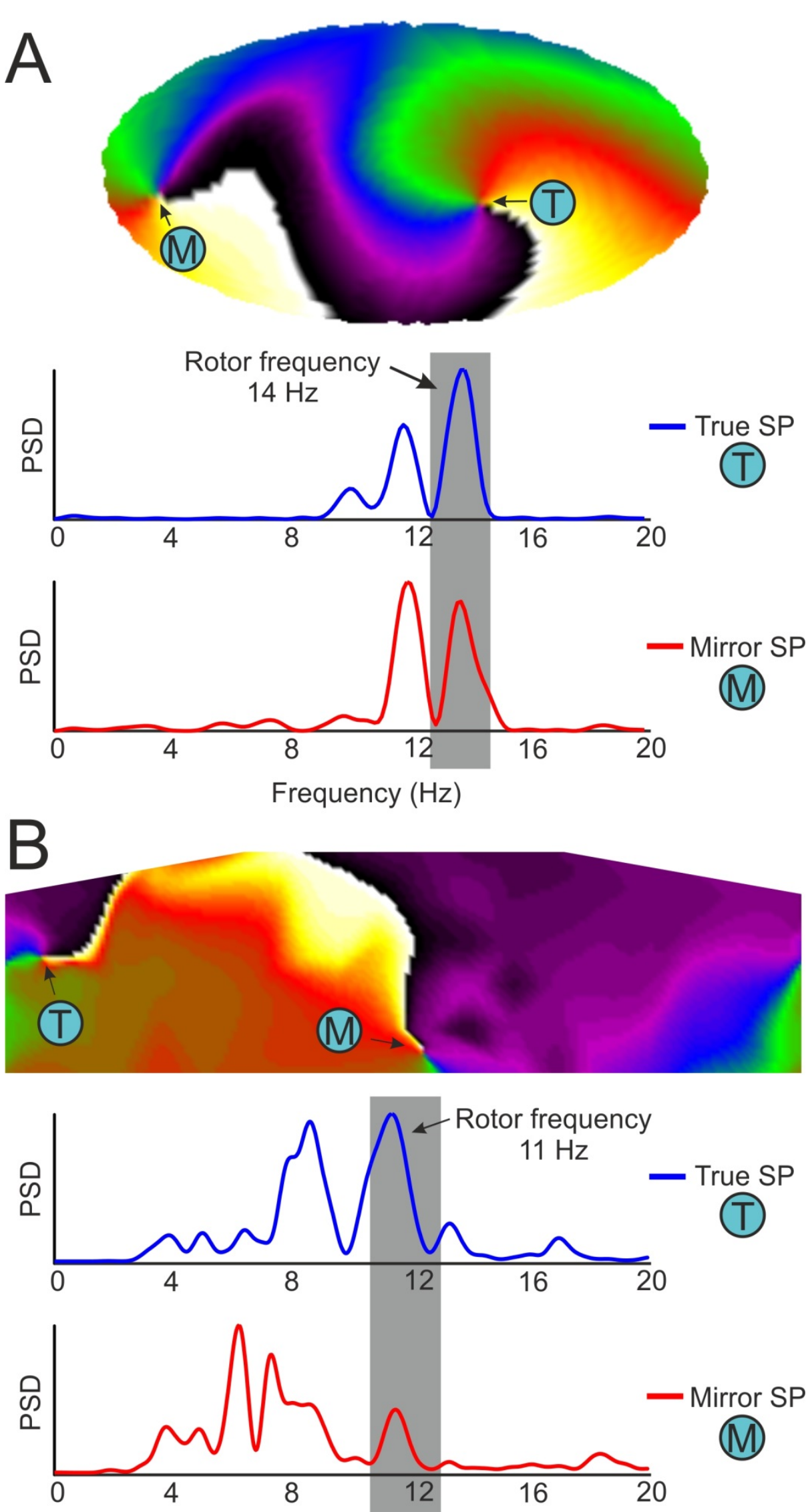

Frequency $(\mathrm{Hz})$ 


\section{FIGURE 5}

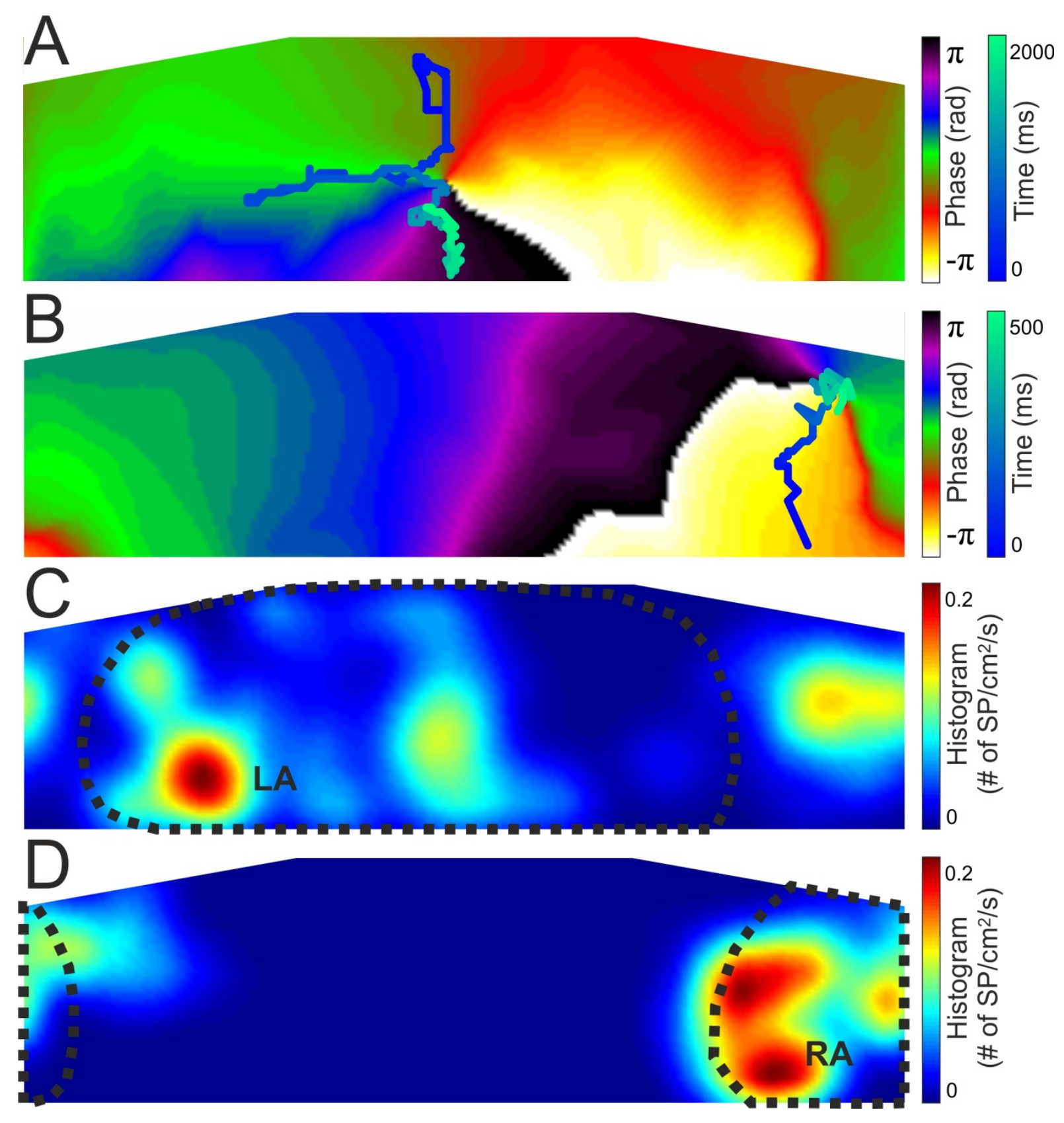

